Restrepo Ruiz, A. L., y Ceballos Ramírez, S. L. (2013). Economía informal en el altiplano del Oriente antioqueño, Colombia. Revista Lebret (5). Bucaramanga, Colombia: Universidad Santo Tomás, pp. 187-210.

\title{
Economía informal en el altiplano del Oriente antioqueño, Colombia*
}

\section{Informal Economy in the Highlands of Eastern Antioquia - Colombia}

Silvia Liliana Ceballos Ramírez ${ }^{1}$ Alba Lucía Restrepo Ruiz²

\section{Resumen}

La economía informal se ha convertido en una importante fuente de recursos para muchos colombianos y habitantes de esta subregión, lo que contribuye a reducir las estadísticas de desempleo. Ante este hecho, surge el interrogante: ¿Para las personas que desarrollan actividades enmarcadas en la economía informal, se generan niveles significativos en la calidad de vida o simplemente subsisten? Para la realización de este estudio se desarrolló una investigación exploratoria y concluyente, utilizando como herramientas un cuestionario estructurado, entrevistas y matrices, para concluir que la calidad de vida de las personas que se dedican a este tipo de actividades es baja, puesto que a menudo no tienen cobertura de un sistema de seguridad social y los ingresos son insuficientes para cubrir incluso sus necesidades básicas.

\section{Palabras clave}

Economía Informal, Empleos, Informalidad, Ingresos.

\section{Códigos de Clasificación JEL: D63, E24, E26, J81.}

\begin{abstract}
The informal economy has become an important resource for many Colombians and people of the subregion, helping to reduce the unemployment statistics. But the question arises: Do people who carry out activities within the informal economy generate significant levels in their quality of life or do they just survive? For this study, we developed an exploratory and conclusive research using a structured questionnaire, interviews and matrices, to conclude that the quality of life of people who engage in this type of activity is low, since they often do not have a social security coverage; income is insufficient to even meet their basic needs.
\end{abstract}

\section{Keywords}

Informal economy, Employment, Informality, Income.

* En este trabajo se presentan los resultados de la investigación Economía Informal en los municipios del Altiplano del Oriente Antioqueño realizado con el apoyo de los estudiantes de los semilleros GRINCOMEX y Pensamiento Económico del programa de Comercio Exterior de la Universidad Católica de Oriente, Colombia.

1 Magíster en Administración, Universidad EAFIT. Coordinadora e Investigadora del Programa de Comercio Exterior de la Universidad Católica de Oriente, Colombia. Correo electrónico: lceballos@uco.edu.co.

2 Magíster en Gobierno, Universidad de Medellín. Profesora Universidad Católica de Oriente, Colombia. Correo electrónico: arestrepo@uco.edu.co. 


\section{La economía informal}

En las décadas de los 50 y de los 60 del siglo pasado, se acudió al concepto de marginalidad para caracterizar las poblaciones migrantes del sector rural que ofrecían su trabajo en centros urbanos. La marginalidad socio-laboral que plantea Farias (2012) se caracteriza por una dimensión normativa y una material y se refiere no sólo a la falta de trabajo, sino a la carencia de servicios (Faria, 1976).

Los estudios sobre la informalidad en América latina y el Caribe se remontan a la década de los 70 de ese mismo siglo, con el estudio realizado por el Programa Regional del Empleo para América Latina y el Caribe (PREALC) en Paraguay. A partir de ahí, se comienza a utilizar el término y, se caracteriza como una forma de producción conformada por un conjunto de unidades productivas sin acceso a capital y que asume un alto riesgo empresarial. (PREALC, 1993).

En la literatura económica, se observan varios enfoques sobre la economía informal. El enfoque estructuralista presenta la informalidad como una alternativa frente a la falta de posibilidades de la economía formal; en los abordajes dinámicos se muestra cómo las actividades capitalistas crean una configuración para otras actividades informales, creando espacios que pueden ser ocupados por pequeños productores y trabajadores autónomos. Los espacios informales aparecen en esta última perspectiva, subordinados a los movimientos de producción formal y, de esta forma, nacen, se desarrollan y mueren A su vez, el enfoque regulacionista considera que el sector informal está integrado por un conjunto de actividades que no cumplen con la reglamentación existente, ya sean laborales, fiscales, etc. Investigadores como Bouton, Portes y Castells (citados por Grupo de Investigación Economía Social, GIES, 2005) postulan la existencia del sector informal como resultante de la subordinación del trabajo al capital para mantener el nivel de rentabilidad.

En Colombia se han realizado diversos estudios analizando el mercado laboral informal; unos autores lo han enfocado desde la perspectiva de la segmentación laboral, otros desde las empresas informales y otros se han interesado en la rigidez del mercado laboral y su relación con la informalidad. Entre los autores que estudian la informalidad desde la perspectiva de la segmentación se encuentra Flórez (2002) cuya hipótesis consiste en que el mercado de trabajo se caracteriza por la existencia de dos sectores uno formal, con remuneración adecuada y otro informal, con baja remuneración. Uribe, Ortiz y Correa (2004) realizaron estudios sobre la decisión de estar empleado en el sector informal entre 1988 - 2000, estimando la probabilidad de permanecer en dicho sector, encontrando que la educación es la única variable negativa sobre el empleo informal. Santa María y Rozo (2009), utilizando el Censo empresarial de Cali y Yumbo de 2005, establecieron la incidencia de la informalidad de las empresas de cualquier tamaño, concluyendo que en las microempresas la informalidad es un fenómeno generalizado y que en la mayoría de los casos, tiende a desaparecer con el crecimiento de las empresas. Desde la perspectiva de la rigidez 
del mercado laboral en Colombia, Núñez (2002) plantea una relación positiva entre la informalidad y la evasión fiscal, en particular establece que el impuesto a la renta incentiva a los individuos a participar en el sector informal. De otra parte, Mondragón, Peña y Wills (2009) encontraron que los costos laborales no salariales y el incremento del salario mínimo tienen efectos sobre el tamaño del sector formal e informal. Bernal (2009) encontró que no todos los empleos informales son precarios ni todos los empleos formales son de mejor calidad; además, este es un fenómeno extendido en las zonas urbanas y se encuentra el nivel de satisfacción de estas personas con el empleo que realizan.

En el Departamento de Antioquia se han realizado algunos estudios sobre el mercado laboral informal. Uno de ellos fue el realizado por el Grupo de Investigación de Economía Social (GIES), denominado Apuntes sobre la economía informal: Caso Medellín, en el cual se hace una revisión de las metodologías empleadas para medir la economía informal y se propone una alternativa de medición precisa de este fenómeno, a la vez que se realiza una descripción cualitativa de su incidencia en este municipio (Grupo de Investigación Economía Social, GIES, 2005). En el estudio de Blandón (2011) Territorialización, control y política en el centro de Medellín a comienzos del siglo XXI, se analiza el proceso mediante el cual las mercancías transadas en un mercado globalizado van a parar en grandes centros comerciales, pero también a las calles para el intercambio en mercados minoristas de microescala, transformando la vida social de la mayoría de las ciudades del mundo.

La economía informal tiene tantos desarrollos conceptuales que apuntan a su definición como nombres para llamarla. Sobre la noción del sector informal, algunos autores coinciden en señalar que se inició con el trabajo de Keith Hart, titulado Informal income opportunities and urban employement in Africa en 1971, en el seminario de desempleo presentado en dicho país (Ochoa Valencia, 2004). A partir de la misión de la Organización Internacional del Trabajo (OIT) en Kenia, la informalidad fue definida como una forma urbana de hacer las cosas cuya marca distintiva incluye: pocas barreras a la entrada para el empresario en términos de habilidades y capital requerido, empresas de propiedad familiar; operación en pequeña escala, producción de trabajo intensiva con tecnología adaptada y un mercado no regulado y competitivo (OIT, 1972). Según Gilberto Arango Londoño (2005, p. 474) todas las actividades de producción de bienes y servicios y las transacciones internas y externas de un país que no son registradas oficialmente en las cuentas nacionales ni en ningún otro indicador de la economía de un país pertenecen a la actividad económica informal. En otra de sus definiciones del autor precitado, la economía informal se concibe como la actividad económica no ilegal oculta por razones de elusión fiscal o de control administrativo 3 . En Colombia, el Departamento Administrativo Nacional

3 Informe IV (1) OIT - Trabajo decente para los trabajadores domésticos, Ginebra, 2010, ISBN 978-92-2-321-886, 2010 
de Estadística (DANE) (2011), define como pertenecientes al sector informal de la economía a los ayudantes de familia a los cuales no se les paga un salario, a las empleadas del servicio doméstico, a los trabajadores que se encuentran trabajando por su propia cuenta en actividades propias o familiares (excluyendo trabajadores y técnicos) y a los obreros y empleados asalariados del sector privado y patrones o empleadores vinculados a empresas con diez o menos personas ocupadas.

Sobre lo anterior, Castaño (2000) diferencia las actividades formales de las informales teniendo en cuenta la existencia (o no) de normas de regulación o reglamentaciones legales. Según el mismo autor, generalmente este tipo de actividades no cumplen con ciertas características económicas y administrativas propias de una economía formal, por ejemplo no utilizan tecnologías complejas ni formas avanzadas de producción, no tienen una división del trabajo establecida, no están constituidas jurídicamente como las empresas modernas y tienen distintos tipos de relaciones laborales al mismo tiempo. Una de las características que hace que una determinada actividad pertenezca al sector informal es la de tener una escala reducida; es decir, que las personas o empresas no manejan una producción en gran escala o una gran cantidad de recursos, además no existen muchas barreras para entrar al sector informal. Igualmente, la propiedad de los negocios o empresas es de una sola persona o familia, y en algunos casos no se cumple con reglamentaciones sobre salarios, impuestos, salud y limpieza, normas de construcción y demás. Muchas veces este tipo de actividades no son registradas por los censos o las encuestas, razón por la cual según el autor que estamos reseñando, el sector informal es muy difícil de medir, siendo más común en los países en vía de desarrollo que en los países industrializados. Según Sara Ochoa León (2005) la existencia de economía informal se ha intentado explicar por la insuficiente creación de empleos formales producto del bajo crecimiento del PIB, por la subcontratación de empresas informales por parte de las empresas formales, y por las excesivas regulaciones que aumentan el costo de la formalidad, por ejemplo, por los trámites y recursos necesarios para iniciar un negocio.

Para la medición de la economía informal se utiliza tanto el Método Directo como el Indirecto. El Indirecto permite analizar el comportamiento de variables que sirven como indicadores de las actividades informales, se mencionan dentro del mismo el método de insumo físico y el enfoque monetario. El Método de Insumo Físico está sustentado en el consumo de energía eléctrica que no está explicada por la actividad de la economía formal. Incluye actividades domésticas, actividades realizadas por uno mismo y servicios no registrados. A su vez, el Método del Enfoque Monetario consiste en estimar una demanda de dinero, ya que se basa en la hipótesis de que las actividades de la economía informal son efectuadas en efectivo. En este sentido, 
se estima una demanda de dinero donde el porcentaje que las monedas y billetes representan del agregado monetario, es función de la carga tributaria, medida por la variable ingresos tributarios como porcentaje del PIB.

En el método directo se parte de una definición muy precisa de las características que deben tener las personas que desempeñan actividades informales y se mide su incidencia mediante encuestas a individuos o empresas. Es el método utilizado en Colombia por el Departamento Administrativo Nacional de Estadística (DANE) (2011) el cual clasifica las actividades y unidades informales por sectores de actividad económica.

En nuestro país, las cifras muestran un importante porcentaje de la poblaciónque no se considera desempleada porque ejerce actividades económicas remuneradas periódicamente - y que pertenecen a este sector informal-. Según cifras del DANE, a pesar del mejor ambiente económico del país en los últimos años que se ve reflejado en un aumento importante del Producto Interno Bruto, la economía informal no deja de crecer, ya que cada vez son más las empresas que buscan la evasión de impuestos, provocando en la población un aumento del empleo informal o lo que es peor un aumento en el 'rebusque' o subempleo. Según información presentada por esta entidad ${ }^{4}$, la tasa de desempleo se ubicó en 11,6 mientras que la cifra del empleo informal llegó a 32,7\%. Lo que es aún más preocupante, es que las estadísticas presentadas por esta institución reflejan que los jefes de hogar representan el 32,8\% del total de las personas subempleadas en el país.

Si se hace un análisis por ciudades, de acuerdo a las cifras del DANE, se encuentra que Riohacha es la capital con mayor índice de informalidad, con el 45,7\%, seguida de Sincelejo con el $43,6 \%$ y luego Cali con el $42,1 \%$ y por su parte, las ciudades que tienen menores tasas de subempleo son Bogotá con el 5,7\%; Barranquilla, con el 15,1\% y Quibdó con el 18,8\%. Según rama de actividad, la población ocupada informal en las trece áreas metropolitanas se encontraba clasificada principalmente en el subsector comercio, hoteles y restaurantes (40,7\%). Según posición ocupacional, los trabajadores por cuenta propia constituyen el 58,9\% de la población ocupada informal. Al observar los niveles educativos, se detecta que el 52,4\% de la población ocupada en el empleo informal tenía nivel educativo secundario mientras el 30,4\% tenía sólo había cursado la educación primaria. El principal lugar de trabajo de la población ocupada informal en el total de las trece áreas metropolitanas del país fue un local fijo (32,3\%). Respecto a la seguridad social, se encontró que del total de ocupados en el sector informal sólo el 41,9\% tenía seguridad social. Del total de la población informal, 10,1\% tenía seguridad social.

4 Los datos son del año 2010, año en que se recogió la información para el estudio. 
Tabla 1. Empleo Informal

Proporción de informalidad y población de ocupados en el empleo informal

Total 13 áreas metropolitanas

Trimestre móvil mayo - julio (2009-2010)

\begin{tabular}{lcccc}
\hline \multicolumn{1}{c}{ Total 13 áreas } & \multicolumn{2}{c}{ Proporción } & \multicolumn{2}{c}{$\begin{array}{c}\text { Población ocupada informal } \\
\text { (miles) }\end{array}$} \\
\hline & May - Jul 09 & May - Jul 10 & May - Jul 09 & May - Jul 10 \\
\hline $\begin{array}{l}\text { Población ocupada en el empleo } \\
\text { informal }\end{array}$ & 52,4 & 51,9 & 4673 & 4761 \\
\hline
\end{tabular}

NOTA: Datos expandidos con proyecciones de población, elaboradas con base en los resultados del Censo 2005.

Fuente: DANE, Gran Encuesta Integrada de Hogares - GEIH

Figura 1. Empleo Informal
Proporción de la población ocupada en el empleo informal
Total 13 áreas metropolitanas
Trimestre móvil mayo - julio $(2007-2010)$

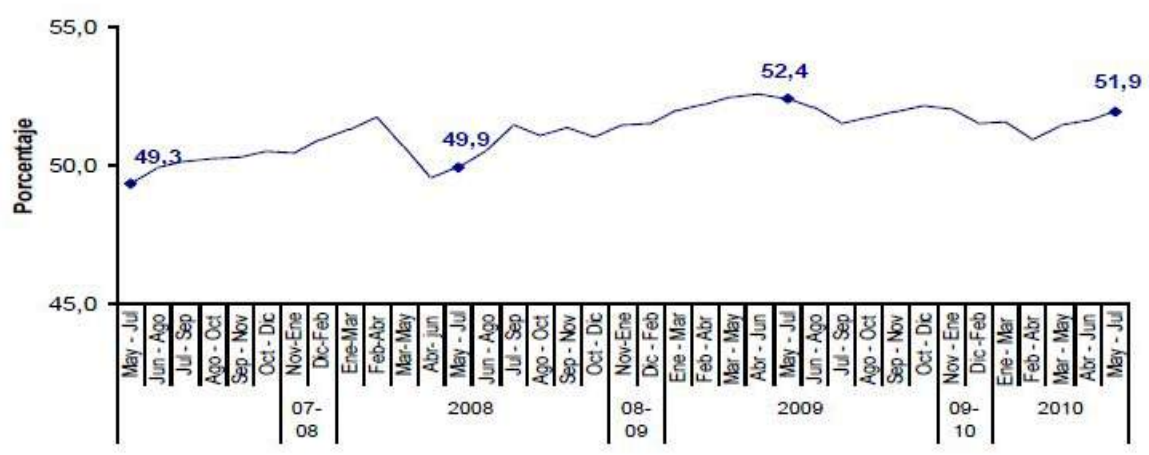

Fuente: DANE, Gran Encuesta Integrada de Hogares - GEIH 
Figura 2. Ocupados informales

Población ocupada en el empleo informal

Total 13 áreas metropolitanas

Trimestre móvil mayo - julio $(2007-2010)$

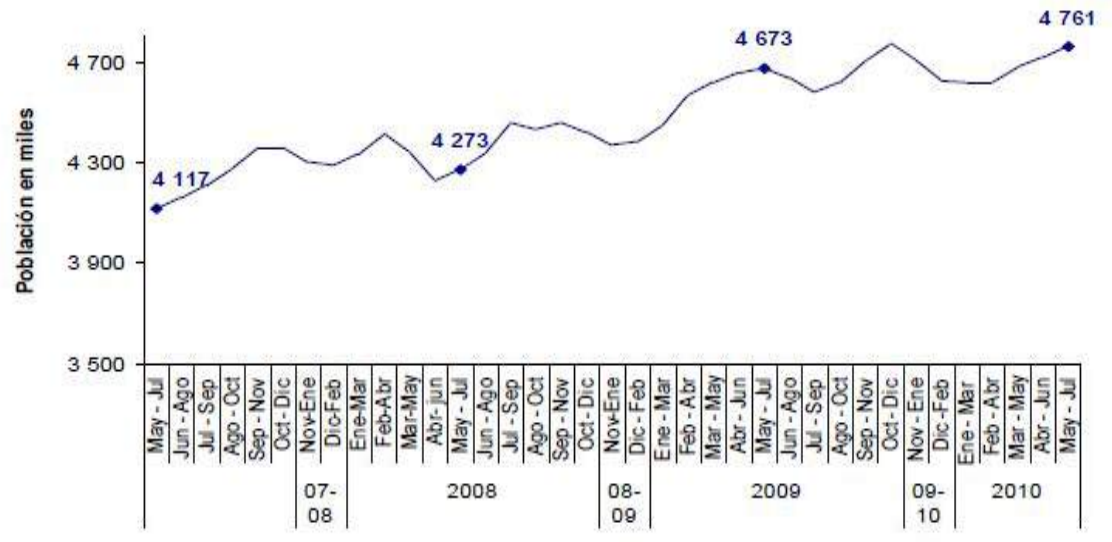

Fuente: DANE, Gran Encuenta Integrada de Hogares - GEIH

\section{El departamento de Antioquia}

Antioquia es un departamento de Colombia localizado al noroeste del país. Ocupa un territorio de unos $63.000 \mathrm{~km}^{2}$ siendo el sexto departamento más extenso de Colombia y el $2^{\circ}$ más poblado, tras el distrito capital de Bogotá. Tiene 125 municipios agrupados en nueve subregiones y su capital es la ciudad de Medellín.

Las subregiones en las que se encuentra dividido el departamento son: El Bajo Cauca, Magdalena Medio, Nordeste Antioqueño, Urabá, Occidente Antioqueño, Suroeste Antioqueño, Valle de Aburra, Norte Antioqueño y Oriente Antioqueño. En este último se encuentra la subregión de Altiplano que es objeto de este estudio y está conformada por los siguientes municipios: Carmen de Víboral, El Retiro, Santuario, Guarne, La Ceja, La Unión, Marinilla, Rionegro y San Vicente 


\section{Mapa 1. Subregiones del Departamento de Antioquia}

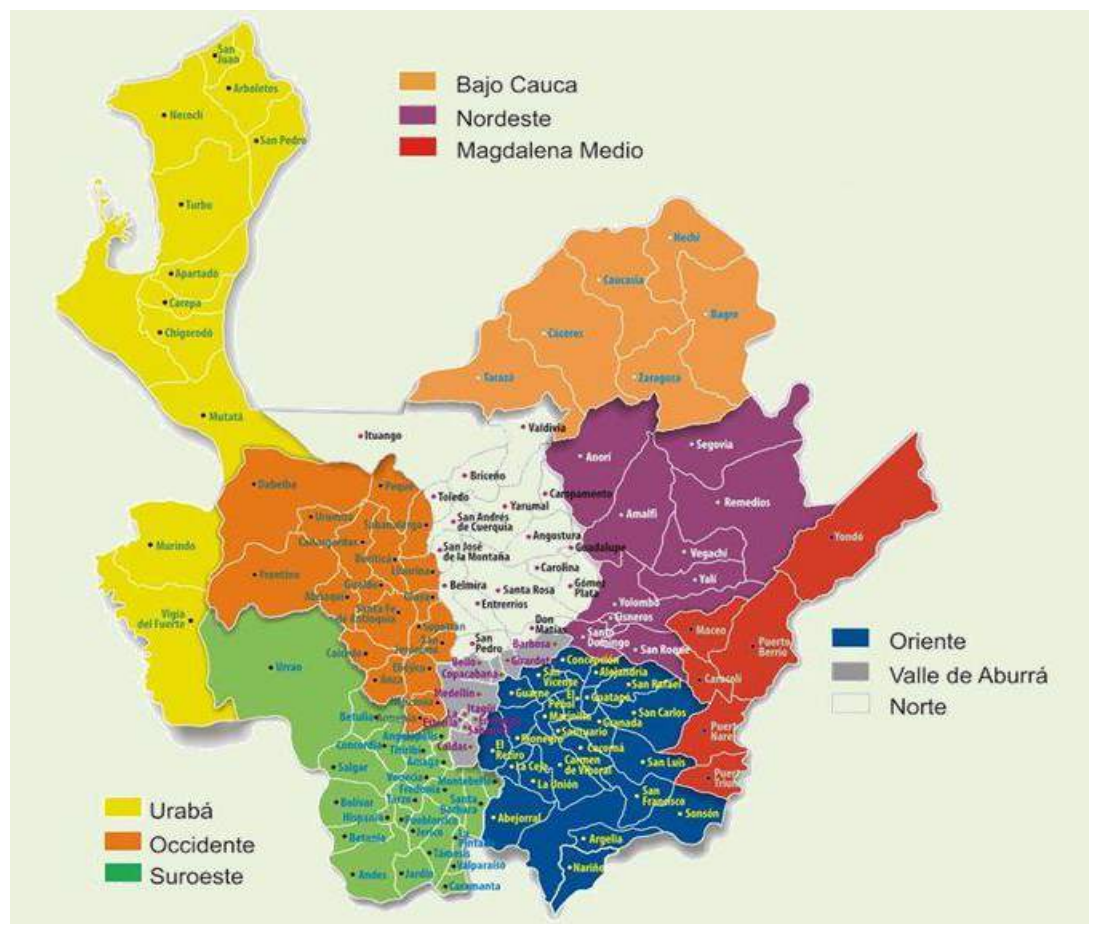

Fuente:http://www.google.com.co/imgres?imgurl=http://sanluis-antioquia.gov.co/apc-aa-fil es/64343634626563663363383235333038/Mapa_San_Luis003.jpg\&imgrefurl=http://sanluis-antioquia. gov.co.

\section{Oriente Antioqueño}

El Oriente Antioqueño tiene una extensión de 7021 km², está conformado por 23 municipios del Departamento de Antioquia ${ }^{5}$, los cuales se han agrupado en varias zonas $^{6}$. Esta zona se ha considerado como la despensa agrícola departamental ${ }^{7}$ por la producción de frutas y hortalizas, además, esta región se proyecta como

5 Para algunos entes, la región del Oriente Antioqueño está conformado por 25 municipios, agregando a la subregión de embalses San Roque y Santo Domingo.

6 La información de los párrafos anteriores hace parte del Estudio de diagnóstico contextualización de los 23 municipios del oriente antioqueño sobre la situación del conflicto político armado, los derechos humanos, el derecho internacional humanitario, las organizaciones sociales y la gobernabilidad democrática, realizado por la Unidad de análisis observatorio de paz y reconciliación del Oriente Antioqueño.

$7 \quad$ La subregión aporta el 60\% de los productos agropecuarios del Departamento. 
un eje básico para el desarrollo regional del departamento de Antioquia, lo que se manifiesta con la planeación y el desarrollo de diferentes proyectos de inversión. La economía de muchos de sus municipios ha sido fundamentalmente agrícola, pecuaria y forestal, las fuentes de empleo en el casco urbano son escasas, en muchos de ellos esas oportunidades las ofrecen las entidades públicas y el sector cooperativo. En el ámbito rural, sólo se cuenta con los jornales pagados por la recolección de cultivos en épocas de cosecha, sobresaliendo la yuca, plátano, maíz, café, fríjol, guayaba, caña de azúcar, cacao, maderas. La economía agrícola es de subsistencia, pues no quedan excedentes para la comercialización a gran escala. La presencia de cultivos de uso ilícito como la coca, sumado a los laboratorios para su procesamiento, hace de la economía de algunos de los municipios del Oriente Antioqueño sea su principal actividad económica, lo cual se convierte en una amenaza para el medio ambiente y los cultivos pan coger.

\section{Metodología}

Para la realización de este estudio se desarrolló una investigación exploratoria consultando fuentes secundarias, y se hicieron entrevistas a algunas personas conocedoras del tema. Luego de haber completado esta fase, se procedió a desarrollar la parte concluyente, en la cual se utilizó el método directo, y se aplicó un cuestionario estructurado que contaba con algunas preguntas que se encuentran en el módulo de informalidad de la Encuesta Continua de Hogares (ECH), que aplica el DANE en las principales ciudades del país y otras diseñadas con el objeto de obtener información sobre los niveles de calidad de vida de las personas que desarrollan actividades enmarcadas en la economía informal. Una vez elaborado el cuestionario, se hizo una prueba piloto para ver si la redacción de las preguntas era clara, esto permitió hacer ajustes y modificaciones. El método para diligenciar el cuestionario y captura de datos fue el de entrevista personal.

El estudio se centró en personas que desarrollaran actividades de la economía informal de los municipios de Rionegro, La Ceja, Guarne y El Retiro, Marinilla, El Carmen de Víboral, El Santuario, La Unión y San Vicente.

A continuación se presenta una Tabla explicativa con las características de la población: 
Tabla 2. Características de la población objetivo

\begin{tabular}{cc}
\hline $\begin{array}{c}\text { Partes integrantes de una } \\
\text { definición de población }\end{array}$ & Descripción \\
\hline Elemento & $\begin{array}{c}\text { Personas dedicadas a alguna actividad enmarcada } \\
\text { como economía informal }\end{array}$ \\
\hline Extensión & $\begin{array}{c}\text { Municipios de rionegro, la ceja, guarne y } \\
\text { el retiro, marinilla, el carmen de víboral, el } \\
\text { santuario, la unión y san vicente }\end{array}$ \\
\hline Tiempo & Entre diciembre de 2010 y febrero de 2011 \\
\hline
\end{tabular}

Fuente: autores.

Para determinar el tamaño de la muestra se empleó la fórmula de poblaciones infinitas con un universo de 378343 habitantes de la subregión, con un nivel de confianza del $95 \%$, un margen de error del $5 \%$ y un intervalo de confianza de $2.18 \%$, dando como resultado la elaboración de 384 encuestas. Pero al poder contar con varias personas para la elaboración del trabajo de campo se realizaron 951 encuestas, que se repartieron en los 9 municipios objeto de estudio, generándose un intervalo de confianza de $1.38 \%$. Luego se procedió a repartir el número de encuestas asignadas a cada por municipio, de acuerdo a las posibles actividades informales que se desarrollan en cada uno de ellos. A partir de la información obtenida, se procedió a hacer un análisis de la situación particular de la economía informal en cada uno de los municipios.

Tabla 3. Población

\begin{tabular}{ccc}
\hline Población & Censo 2005 & Proyección 2010 \\
\hline Rionegro & 100502 & 110329 \\
\hline La Ceja & 46933 & 49523 \\
\hline La Unión & 17842 & 18564 \\
\hline El Carmen & 41012 & 43825 \\
\hline El Retiro & 16976 & 18081 \\
\hline Guarne & 39541 & 43576 \\
\hline San Vicente Ferrer & 19438 & 18330 \\
\hline Santuario & 26287 & 26754 \\
\hline Marinilla & 45548 & 49361 \\
\hline TOTAL & $\mathbf{3 5 4 0 7 9}$ & $\mathbf{3 7 8 3 4 3}$ \\
\hline
\end{tabular}

Fuente: www.dane.gov.co 


\section{Resultados de la investigación}

Uno de los resultados más llamativos del estudio se refiere a la constatación de que el género no influye para la realización de actividades informales; en casi todos los municipios se da una proporción casi de 50 - 50. En La Ceja se encontró que el $62 \%$ de las personas que se dedican a labores enmarcadas en la informalidad eran hombres y, en El Retiro se detectó que el 64\% es desarrollado por las mujeres.

Tabla 4. Género por municipio

\begin{tabular}{|c|c|c|c|c|c|c|c|c|c|c|c|c|c|c|c|c|c|c|}
\hline \multirow[b]{2}{*}{ ном. } & \multicolumn{2}{|c|}{$\begin{array}{l}\text { MARI- } \\
\text { NILLA }\end{array}$} & \multicolumn{2}{|c|}{$\begin{array}{c}\text { EL } \\
\text { CARMEN }\end{array}$} & \multicolumn{2}{|c|}{$\begin{array}{c}\text { LA } \\
\text { CEJA }\end{array}$} & \multicolumn{2}{|c|}{$\begin{array}{c}\text { SAN } \\
\text { VICENTE }\end{array}$} & \multicolumn{2}{|c|}{$\begin{array}{c}\text { RIO- } \\
\text { NEGRO }\end{array}$} & \multicolumn{2}{|c|}{ GUANE } & \multicolumn{2}{|c|}{$\begin{array}{c}\text { EL } \\
\text { RETIRO }\end{array}$} & \multicolumn{2}{|c|}{$\begin{array}{c}\text { SAN- } \\
\text { TUARIO }\end{array}$} & \multicolumn{2}{|c|}{ LA UNIÓN } \\
\hline & 51 & 0.49 & 57 & 0.54 & 65 & 0.62 & 45 & 0.43 & 48 & 0.46 & 50 & 0.46 & 38 & 0.36 & 62 & 0.58 & 50 & 0.48 \\
\hline MUJ. & 54 & 0.51 & 48 & 0.46 & 40 & 0.38 & 60 & 0.57 & 57 & 0.54 & 59 & 0.54 & 67 & 0.64 & 45 & 0.42 & 55 & 0.52 \\
\hline TOTAL & 105 & 1.00 & 105 & 1.00 & 105 & 1.00 & 105 & 1.00 & 105 & 1.00 & 109 & 1.00 & 105 & 1.00 & 107 & 1.00 & 105 & 1.00 \\
\hline
\end{tabular}

Fuente: autoras a partir del desarrollo de la investigación.

Figura 3. Género por municipio

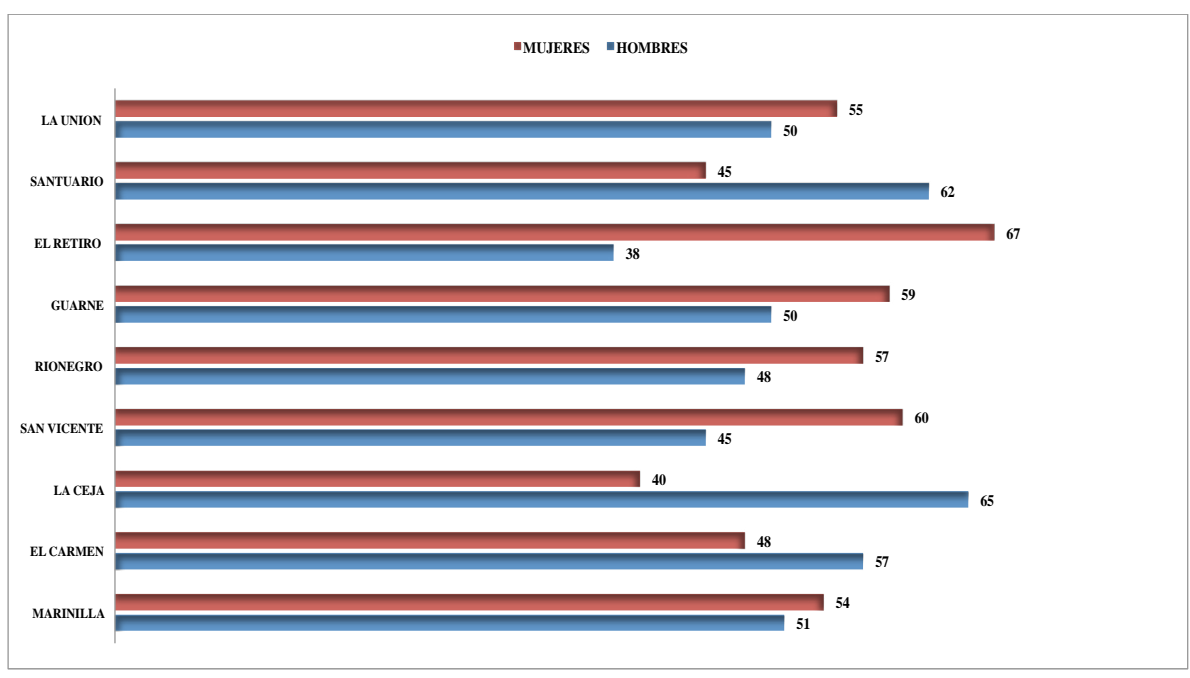

Fuente: autoras a partir del desarrollo de la investigación. 
Las actividades que desarrollan las personas encuestadas son:

- Vendedores ambulantes de ropa, comida, dulces, frutas y verduras, minutos, cachivaches,

- Vendedores de productos por Catálogo, destacándose productos de belleza de Avon, Ebel, Yambal, de dulces y Chocolatería y de ropa interior y exterior.

- Despachadores de carros.

- Agricultores.

- Jornaleros ${ }^{8}$.

- Vendedores estacionarios ${ }^{9}$.

- Vendedores de Rifas, chance y loterías.

- Trabajadoras domésticas por días.

- Trabajadoras sexuales.

- Lustrabotas.

- Modistas.

- Artesanos.

- Bultiador o cargador.

- Trabajadores independientes.

- Cuidadores de niños.

- Mandaderos.

- Carretilleros - cocheros.

- Manicuristas.

- Recicladores.

- Ayudantes de cocina, de construcción.

Ante las pocas oportunidades laborales que se dan en los municipios de la subregión y al no tener otro medio de subsistencia, se observa que las personas ven a las actividades informales como una oportunidad de generar ingresos y poder satisfacer las necesidades que tienen. Un $29 \%$ de las personas encuestadas llevan más de 10 años dedicadas a estas labores; un 14\% ejercen esta labor de 6 a 10 años;

Son trabajadores de campo o de construcción por días.

Estos rotan los productos que ofrecen de acuerdo a las celebraciones de navidad, amor y amistad, madre y padre

Halloween, día de la mujer, día de la Santa Cruz, etc. 
un 19\% entre 3 y 6 años, un $21 \%$ de 1 a 3 años y un 17\% menos de un año, lo cual significa que el fenómeno tiene profundas raíces en las características de la economía regional y que los municipios han demostrado su incapacidad para generar empleos formales que absorban la creciente demanda de la población.

Figura 4. Tiempo dedicado a la actividad

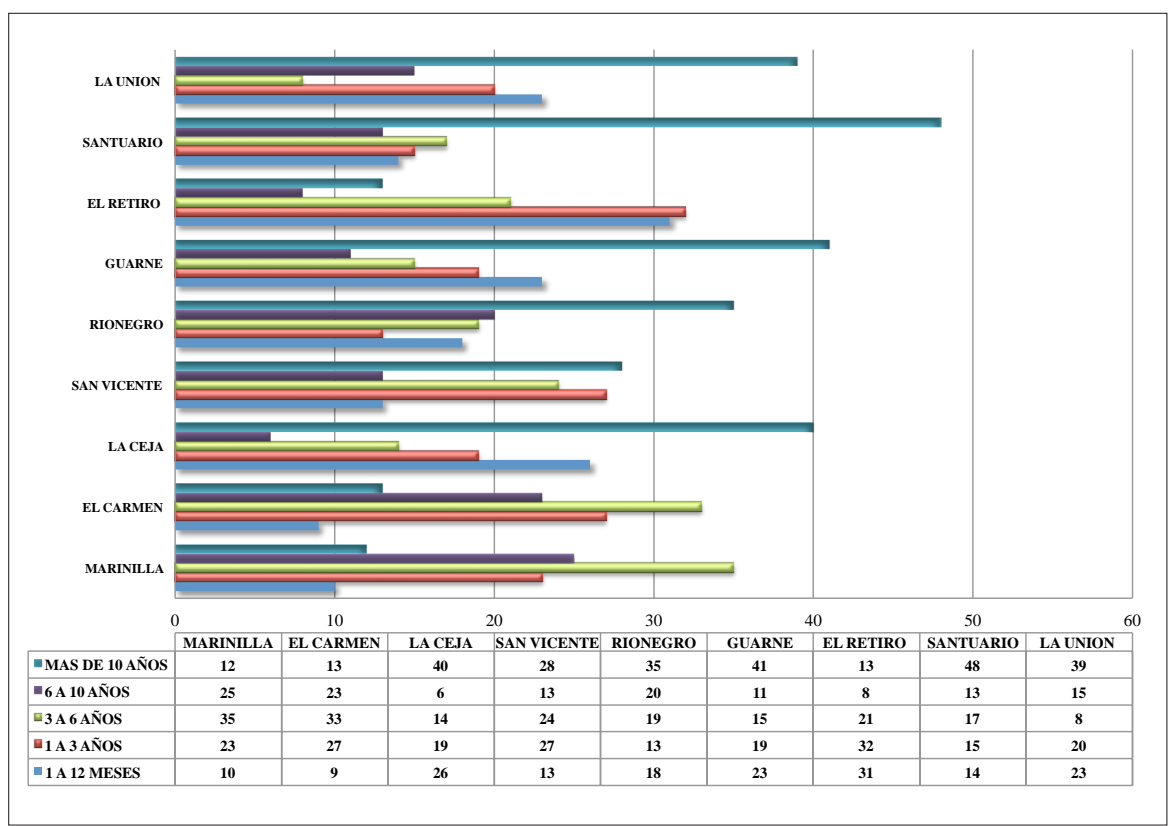

Fuente: autoras a partir del desarrollo de la investigación.

Para el 65\% de la Población encuestada, el desarrollo de las actividades a la cual se dedican en el marco de la informalidad, les ha permitido lograr la Independencia Económica, el 35\% de la población restante considera que los recursos que se obtienen por este medio no permiten alcanzar las metas económicas que se han fijado. Por tanto, es esta franja de la población la que debe ser objeto prioritario de la política pública con el fin de aumentar el nivel de necesidades satisfechas de los habitantes de la región objeto de estudio. 
Figura 5. Actividad que ha permitido la independencia económica

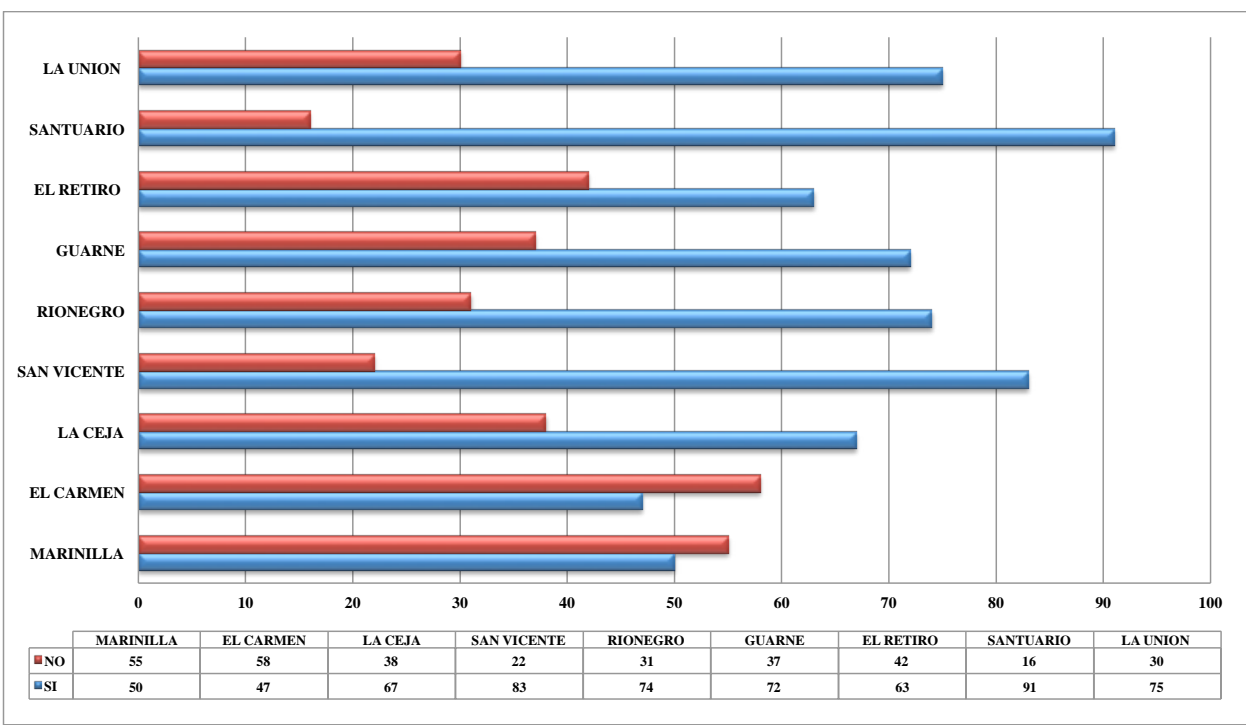

Fuente: autoras a partir del desarrollo de la investigación.

Con la investigación se encontró que en los municipios de Marinilla y El Carmen es donde los encuestados consideran que el trabajo que realizan no les ha permitido esa independencia económica. En los municipios donde hay un alto porcentaje donde las personas encuestadas creen que han logrado la independencia económica son: El Santuario, San Vicente, Rionegro y La Unión.

Los principales motivos por los cuales las personas encuestadas consideran que no han podido lograr la independencia económica se encuentran 222 personas encuestadas (68\%), que consideran que el ingreso que reciben por la actividad desempeñada es muy bajo, 29 personas (8\%), consideran que esos ingresos no alcanzan debido al continuo incremento de los precios, 26 (7.9\%) creen que son actividades inestables, 19 personas $(6 \%)$ tienen muy poco tiempo para dedicarle a esta actividad, 17 personas (5\%), apenas están empezando, y un 5\% (16 personas) consideran que las causas para no lograr la independencia tienen que ver con que el negocio no es propio.

El nivel de ingresos mensuales adquirido por las personas encuestadas, en el desarrollo de estas actividades es menos de un salario mínimo en el 50\% de los casos, un $32 \%$ de los encuestados obtienen un salario mínimo, un $16 \%$ entre 2 y 3 salarios mínimos; un $1 \%$ gana entre 4 y 7 salarios mínimos y un $1 \%$ más de 8 salarios mínimos. 
Figura 6. Nivel de ingresos obtenidos

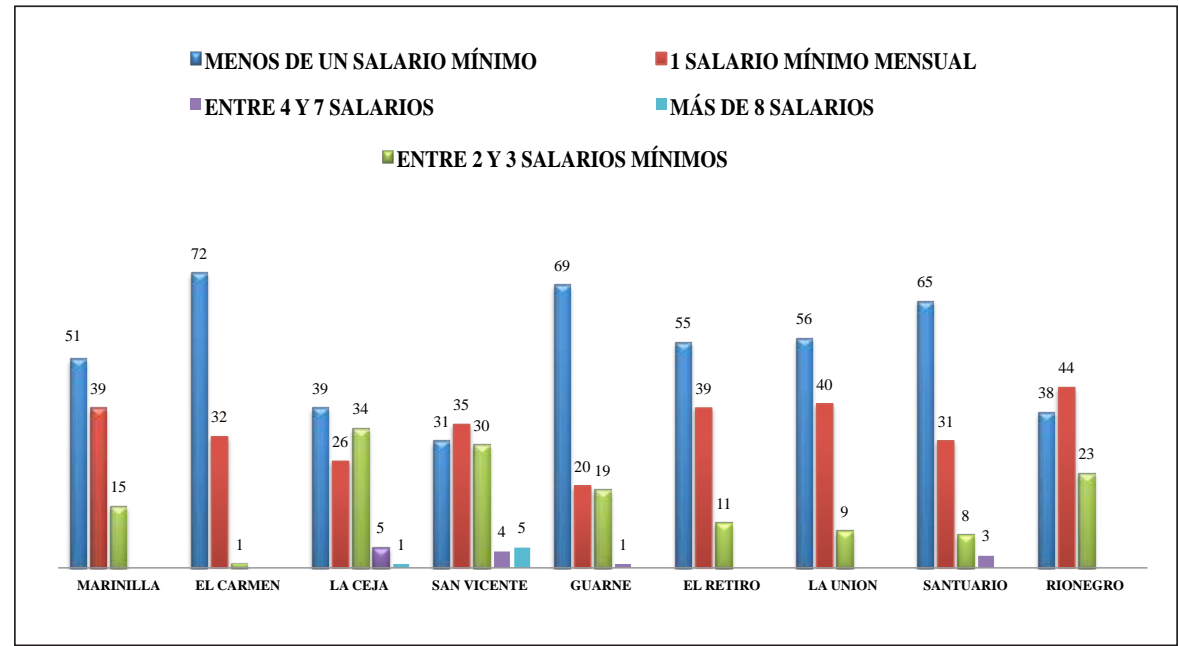

Fuente: autor a partir del desarrollo de la investigación.

En términos de los egresos, un 58\% de ellos se gasta menos de un salario mínimo, un $23 \%$ un salario mínimo, un $15 \%$ entre 2 y 3 salarios mínimos, un $3 \%$ entre 4 y 7 salarios mínimos y un $1 \%$ más de ocho salarios mínimos.

Figura 7. Nivel de egresos

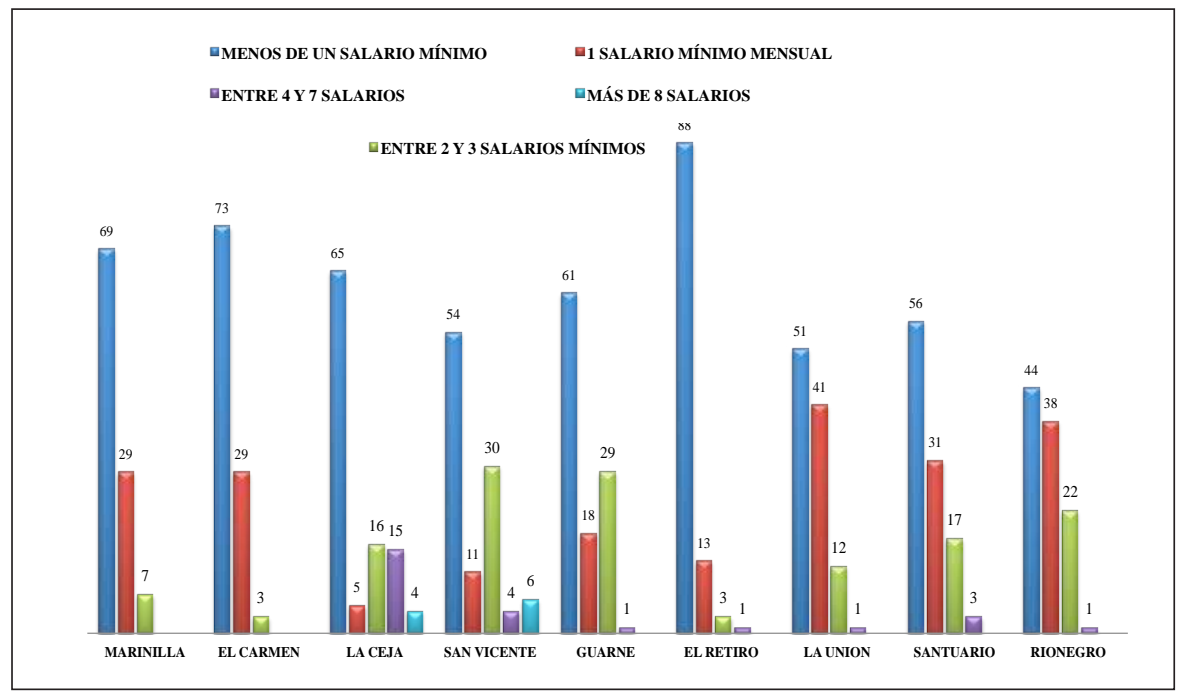

Fuente: autor a partir del desarrollo de la investigación. 
Haciendo un comparativo entre lo que se ganan las personas encuestadas y lo que se gastan, se puede observar que quienes obtienen ingresos menores de un salario mínimo, entre 4 y 7 salarios mínimos y más de 8 salarios se encuentran en déficit para cubrir todas las necesidades.

Figura 8. Relación de ingresos - egresos

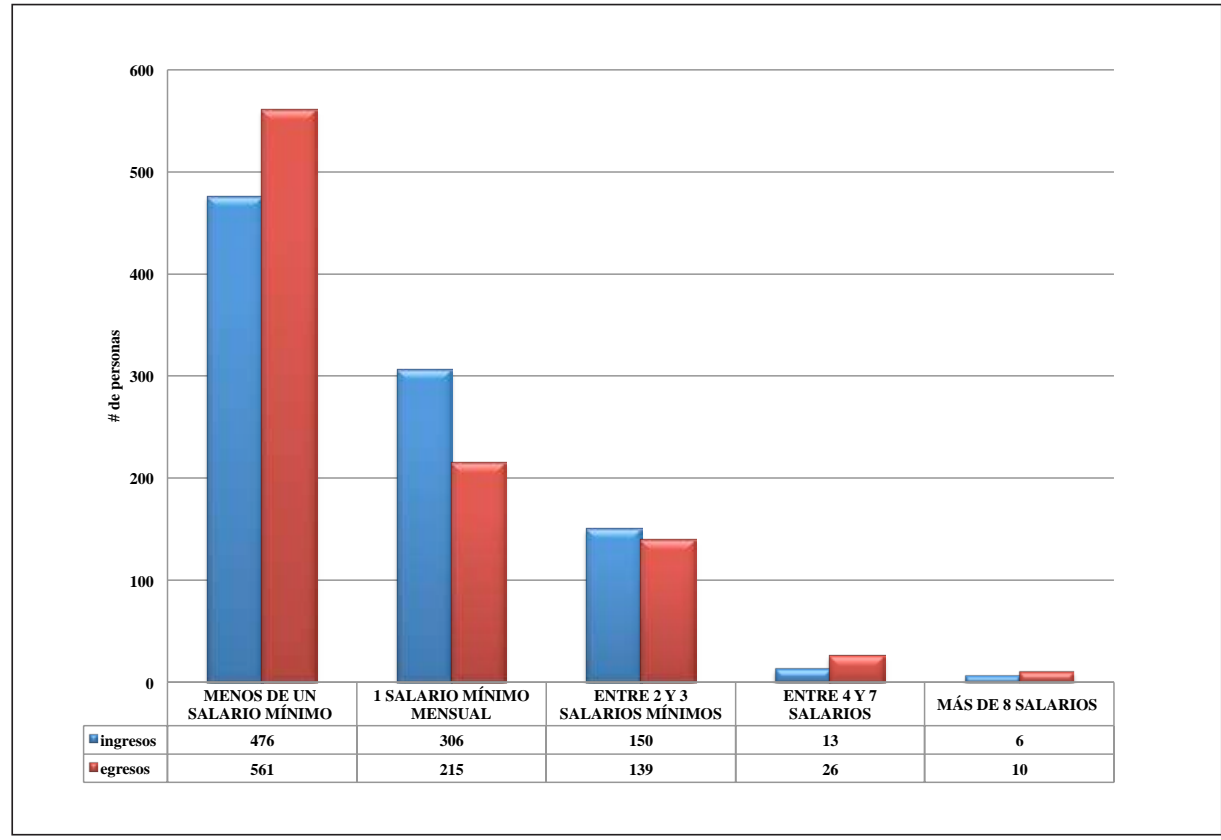

Fuente: autor a partir del desarrollo de la investigación.

En cuanto al tiempo laborado, 485 personas encuestadas (51\%), trabajan siete días a la semana, 187 (20\%) trabajan menos cinco 5 días; 183 (19\%) trabajan seis días y el 96 restante (10\%) laboran cinco días. Los motivos que tienen las 96 personas para laborar menos de 5 días son: a un $57 \%$ solo los contratan ese número de días; un $25 \%$ se dedica a otras actividades y solo le queda unos pocos días para dedicarse a esta actividad independiente y un 18\% trabaja los fines de semana. El $44 \%$ de las personas encuestadas (418) trabajan más de 8 horas al día; un 30\% (288) trabajan las 8 horas al día y un 26\% (245) trabajan menos de 8 horas. 
Figura 9. Cuántos días labora en la semana

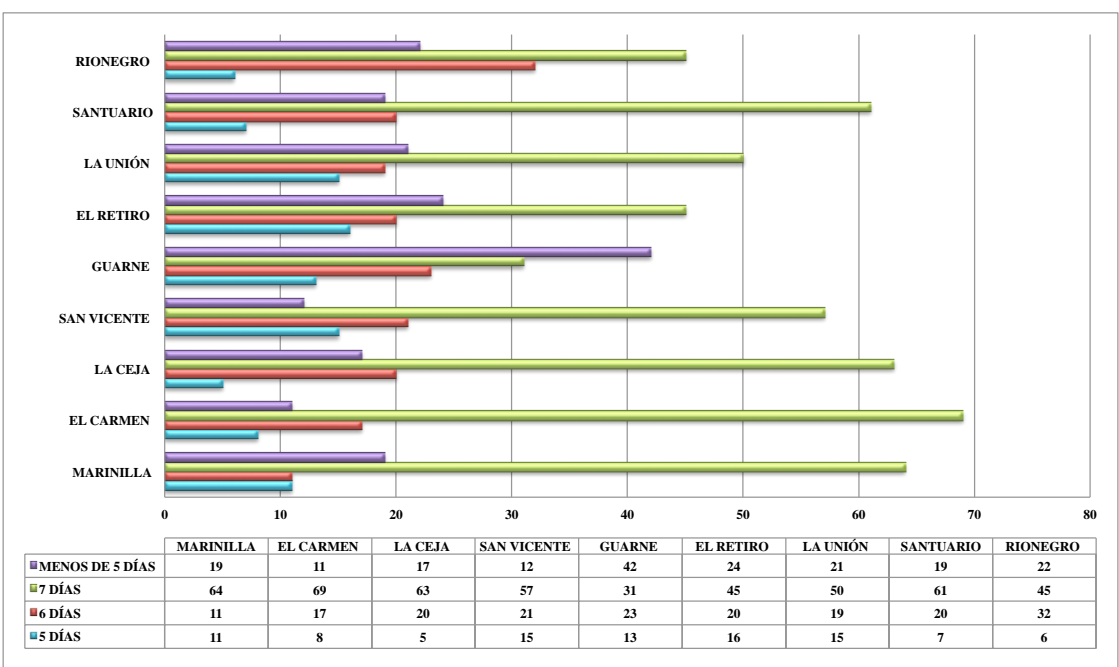

Fuente: autoras a partir del desarrollo de la investigación.

Los motivos que llevaron a las personas encuestadas a dedicarse a la actividad en un $37 \%$ (349) es la independencia que obtienen; un 26\% (247) no consiguió empleo en una empresa, un 17\% (162) está dedicado al hogar y tiene poco tiempo para dedicarle a la actividad que realiza, un $7 \%$ (73) por que fueron despedidos y un $13 \%$ (120) tienen otros motivos. Las 120 personas que tienen otros motivos para dedicarse a la actividad se encuentran la iniciativa propia (13\%), la edad (13\%), deseaban un cambio de actividad (13\%), es lo que sabe hacer (11\%), tiene una discapacidad (11\%), obtiene dinero $(10 \%)$, requiere más ingresos $(7 \%)$, por la crisis económica $(6 \%)$, con esto se ayuda (5\%), tiene tiempo para estudiar (5\%), toda la vida ha trabajado en eso (3\%) y es el negocio de la familia (3\%).

El nivel de satisfacción de 439 personas (46\%) con las actividades que realizan es alto, lo que demuestra el carácter conformista de la población, 438 (46\%) es medio y sólo en 74 de ellos (8\%) es bajo. 623 personas encuestadas (65\%), piensan seguir ejerciendo la actividad, 177 (19\%) piensan buscar un nuevo empleo, 123 (13\%), piensan montar un negocio y, 28 personas (3\%) tienen otras expectativas.

Un 63\% de las personas encuestadas (598) prefieren no trabajar en una empresa, al 37\% restante (353) les gustaría contar con la estabilidad laboral que proporciona la vinculación con el sector formal. Los principales motivos que tienen 353 personas para trabajar en una empresa son para $215(60 \%)$ las prestaciones sociales, para 63 (17\%) las empresas otorgan estabilidad laboral, para $39(11 \%)$ tener un sueldo fijo, para $36(10 \%)$ mayores ingresos. 
Figura 10. Preferiría trabajar en una empresa

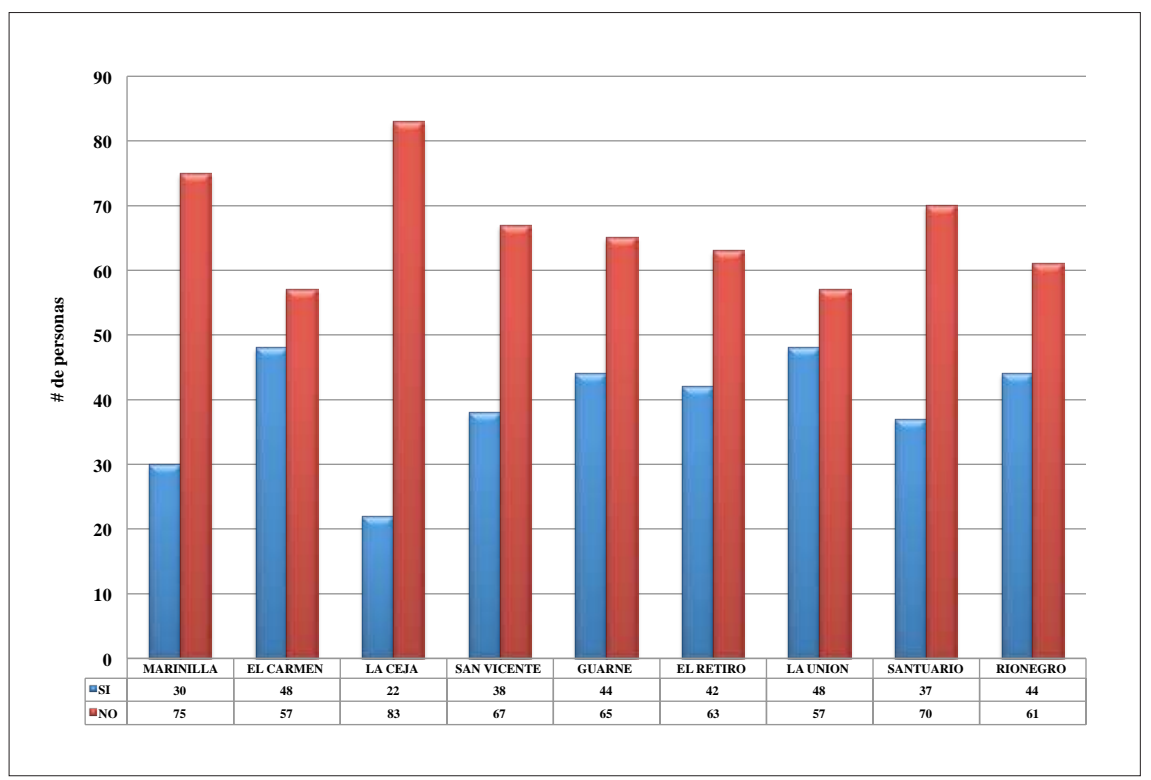

Fuente: autoras a partir del desarrollo de la investigación.

Entre los motivos que tienen las personas para no laborar en una empresa se encuentran: la independencia (47\%), la edad (23\%), no les gusta tener un jefe que los moleste (10\%), desarrollan con éxito otras actividades $(5 \%)$, y el $15 \%$ restante de las personas está dedicado al hogar, tienen alguna enfermedad que los incapacita, no pueden cumplir los horarios en la empresas o no tiene estudios para buscar un mejor empleo. Las principales ventajas que ven las personas encuestadas de ser independiente son: el manejo del tiempo, ser su propio jefe, no hay autoridad por encima de ellos, nadie los maltrata, están pendientes del hogar, tienen su dinero, trabajan cuando desean, trabajan en la casa, y pueden incrementar su salario con mayor trabajo.

Las principales desventajas encontradas por estas personas, es que no cuentan con prestaciones sociales, la presión al no tener en ciertos momentos el dinero que se necesita para pagar las obligaciones que se tienen, la esclavitud que genera el desarrollo de estas actividades, los pocos ingresos, la inseguridad de la calle, la competencia, la incertidumbre y la inestabilidad.

En cuanto al pago de la salud, se encontró que un $66 \%$ de las personas encuestadas (629) no pagan salud y el 34\% restante (322) sí. Un 86\% de los encuestados (819) no 
pagan pensión y, un 14\% (132) sí lo hace. Un 92\% (883) de ellos no tienen cesantías y el $8 \%$ restante han hecho una provisión de sus ganancias para este rubro. En cuanto al pago de servicios funerarios un $57 \%$ de ellos (536) lo hacen y, un $43 \%$ no lo hacen (404). En este punto se encuentran los mayores limitantes de la informalidad que propicia empleos precarios y condiciones de vida limitados, al tiempo que obstaculiza las proyecciones futuras de la población.

Figura 11. Paga salud

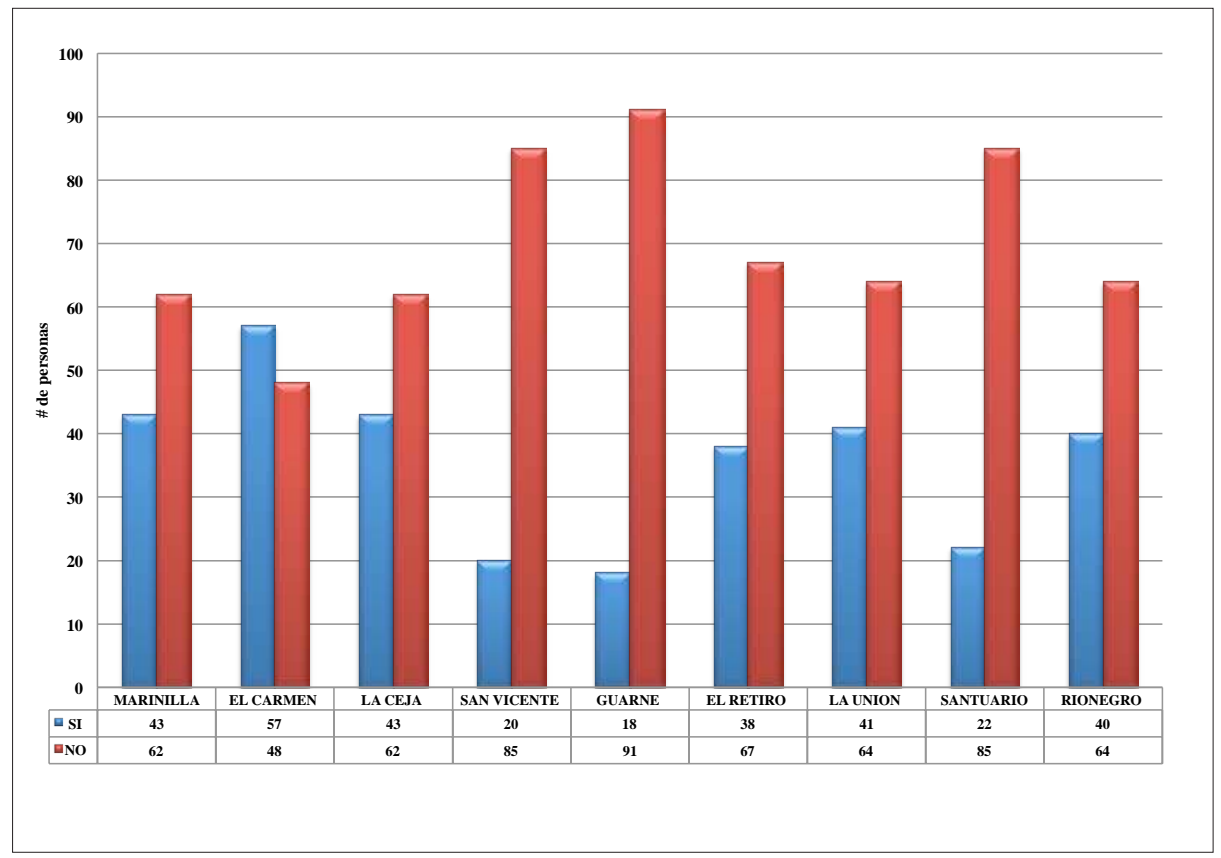

Fuente: autoras a partir del desarrollo de la investigación.

Se encontró que el $61 \%$ de las personas encuestadas no poseen la capacidad de ahorro; un 39\% hace un esfuerzo y guarda para el futuro. 567 personas encuestadas (60\%) no han podido adquirir ningún tipo de bienes desempeñándose en este tipo de actividades. Los bienes que han adquirido 384 de los encuestados se encuentran casa (40\%), electrodomésticos y cosas para el hogar (33\%), carro (16\%), moto (5\%), finca $(2 \%)$, el negocio (2\%), el taller donde trabaja $(1 \%)$ y un lote $(1 \%)$. 
Figura 12. ¿Ahorra?

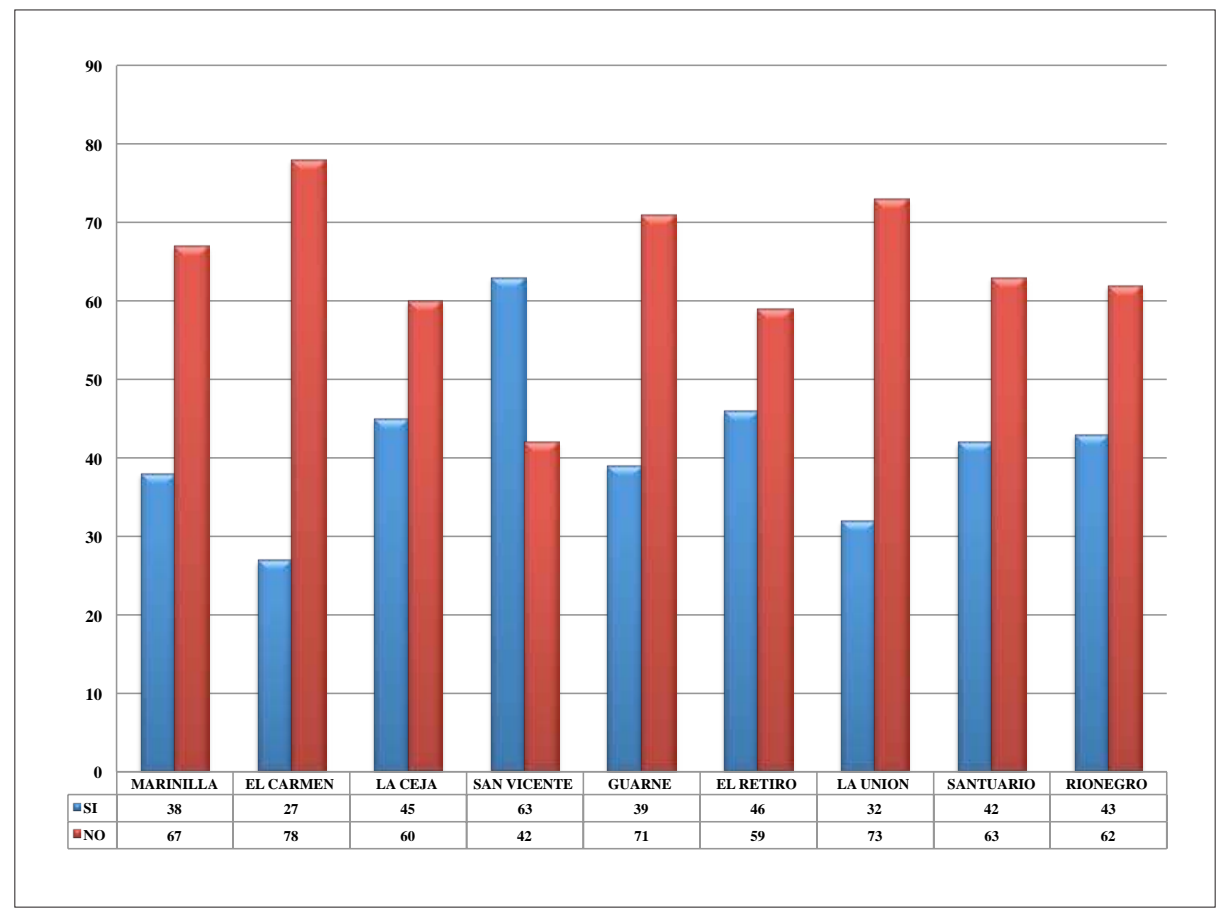

Fuente: autoras a partir del desarrollo de la investigación.

La investigación mostró que un $52 \%$ de los encuestados viven con su cónyuge y sus hijos, un $23 \%$ con los padres y hermanos, un $11 \%$ con sus hijos, un $9 \%$ vive solo y un $5 \%$ con su cónyuge y se puedo determinar cuántas personas dependen económicamente de los trabajadores informales, dando como resultado que un 59\% de los encuestados (556) tienen a su cargo entre 1 y 3 personas; un $25 \%$ (245) no tienen a nadie a su cargo; un 15\% (138) entre 4 y 6 personas dependen del encuestado y un $1 \%$ (12) tiene a su cargo entre 7 y 9 personas.

Cuando las personas encuestadas tienen una necesidad económica recuren en un $36 \%$ a los familiares, un 30\% recurren a los amigos, un $13 \%$ a una entidad financiera, un $12 \%$ a natilleras ${ }^{10}$ y un $9 \%$ recurren a un cuenta gotas ${ }^{11}$.

10 Las natilleras son alternativas de ahorro; estas surgieron durante los años veinte en el Valle de Aburra, consiste en que un grupo de personas se reúnen con el fin de realizar un aporte de dinero según la frecuencia establecida

11 Los cuenta gota o prestamistas gota a gota, es una modalidad de crédito informal, donde la cuota para amortización al capital y los intereses se deben pagar diariamente, las tasas de interés a la que se adquiere al dinero a una tasa diaria del $2 \%$. 


\section{Conclusiones}

La economía informal tiene varias definiciones, pero casi todas convergen a que las actividades aquí incluidas no cumplen con todos los requisitos de ley o con todas las condiciones de calidad que en la economía formal pueden conducir al desarrollo económico. El concepto que se trabajó en la investigación para el análisis de la información es planteado por el DANE como el conjunto de unidades dedicadas a la producción de bienes y prestación de servicios, con la finalidad primordial de crear empleos y generar ingresos para las personas que participan de esa actividad. Estas unidades funcionan típicamente en pequeña escala, con una organización rudimentaria en la que hay muy poca o ninguna distinción entre el trabajo y el capital como factores de producción. Las relaciones de empleo -en los casos en que existan- se basan más bien en el empleo ocasional, el parentesco o las relaciones personales y sociales, no en acuerdos contractuales que supongan garantías formales. Con la definición y de acuerdo a los datos obtenidos con la investigación, se puede decir que la economía informal en los municipios del Oriente Antioqueño no genera desarrollo, sólo ayuda a cubrir medianamente las necesidades básicas de las personas que se dedican a realizarlas, quienes recurren a las prácticas informales por la falta de oportunidades existentes en el sector formal.

En los nueve municipios del altiplano del oriente Antioqueño para el periodo 2010 - 2011, tiempo en que se desarrolló la investigación, la población ocupada informal se encontraba clasificada principalmente en las ramas de comercio, hoteles y restaurantes (80\%) y servicios comunales, sociales y personales (20\%). Según posición ocupacional, la población ocupada informal se encontraba clasificada principalmente en cuenta propia (65\%), seguida por empleado particular (35\%).

La población ocupada en el empleo informal tiene un nivel educativo primario $55 \%$, secundario $15 \%$, educación superior $5 \%$ y el $25 \%$ restante dice que no tuvo estudio. Por tanto, se puede observar que la mayor parte de las personas que pertenecen al sector informal de los municipios encuestados, posee un grado de escolaridad bastante bajo, el cual no supera la educación básica secundaria, lo que se convierte en una desventaja para éstos, ya que disminuyen las probabilidades de emplearse en el sector formal. Esta conclusión que se reitera en la mayoría de los estudios sobre la economía informal, prende las alarmas frente al escaso interés de la política pública por garantizar a la mayoría de la población el acceso a una educación de calidad que les permita competir positivamente en los mercados de trabajo formales.

Muchas de las personas que trabajan en el sector formal tienen la obligación de sostener a una familia y ante la necesidad de conseguir recursos monetarios para su sustento, no tiene otra opción distinta a la de incursionar en el sector informal, y así, hacer parte de ambos sectores o influenciar a un miembro de su familia para que lo haga, con el fin de complementar los ingresos familiares. Los encuestados destinan sus ingresos al pago del arriendo, servicios públicos, educación de los 
hijos y, alimentación; estos rubros consumen gran parte del dinero que se obtienen de la informalidad, dejando al $61 \%$ de los encuestados sin capacidad de ahorro, al $66 \%$ no le alcanza para pagar la cobertura de salud, un $86 \%$ no pagan pensión, un 92\% no tienen cesantías; 694 que representan un $73 \%$ de las personas encuestadas están inscritos en el SISBEN, lo que les genera cierto grado de seguridad en caso de enfermarse o ante una cirugía, un $87 \%$ no les alcanza para el desarrollo de actividades recreativas y un $57 \%$ pagan servicios funerarios.

Las mujeres vienen engrosando las estadísticas del empleo informal en la región; la razón de este fenómeno es que ellas no sólo tienen la responsabilidad de trabajar para complementar los ingresos del hogar, sino que también allí tienen obligaciones, por lo que necesitan horarios flexibles que les permitan hacer las dos cosas a la vez. Estas han visto como la venta de productos por catálogos o alimentos les ha permitido generar ingresos que, aunque dicen que no son muy altos, les permite ayudar con las obligaciones que se van generando en las familias, sostener sus gastos y los de las familias, además tener dinero las hace sentir valiosas. Por tanto, para esta franja poblacional, en muchas ocasiones el sector informal se constituye en la única oportunidad de generar recursos complementarios para el hogar.

Los empleados informales no poseen acceso a créditos de las entidades financieras, ya que no cumplen con los requisitos que estos exigen y no tienen respaldo. Cuando tienen una necesidad recurren a los amigos y familiares, préstamos de natilleras o acuden a los prestamistas cuenta gotas.

El empleo informal genera impactos tanto sociales como económicos. En lo social, porque las personas no cuentan con la seguridad necesaria para gozar de bienestar, ni con apoyo por parte del Estado, y en lo económico, ya que aunque es un factor generador de ocupación para las personas que no ingresan al mercado laboral formal, lo hace en bajas condiciones de calidad, productividad y bajos ingresos.

Un estudio de esta naturaleza se constituye en un punto de referencia, tanto para la acción del Estado como para el diseño de estrategias por parte del sector privado. La política pública debe contemplar, por ejemplo, programas de educación que logren formar a la mayoría de la población en competencias que los capaciten para el trabajo en el sector formal, en tanto que se ofrecen incentivos al sector empresarial para que contribuya con la generación de empleo de calidad. Igualmente, es necesario abrir las opciones para el disfrute de la seguridad social de este segmento de la población que no puede hacerlo a través de los medios convencionales. En épocas en las que la productividad y la competitividad están a la orden del día para jugar positivamente en el concierto internacional, es posible también pensar en encadenamientos productivos promovidos por la empresa privada que utilicen la formalización de las unidades informales como una estrategia válida de innovación empresarial y crecimiento económico que beneficie a ambos lados de la ecuación productiva. 


\section{Referencias}

Arango, G. (2005). Estructura económica colombiana. Bogotá: McGraw-Hill.

Bernal, R. (2009). The informal labor market in Colombia: Identification and characterizacion. Desarrollo y Sociedad, No 63 145-208.

Blandón, M. (2011). Territorialización, control y política en el centro de Medellín a comienzos del siglo XXI. Medellín: Universidad de Antioquia.

Castaño, H. L. (2000). Ensayos sobre economía laboral colombiana. Disponible en: http://www.banrepcultural.org/blaavirtual/economia/ensayoecono/indice.htm

Ochoa, D. (2004). Informalidad en Colombia. Causas, efectos y características de la economía del rebusque. Estudios Gerenciales No 90 Universidad ICESI, Cali

Departamento Administrativo Nacional de Estadística (DANE). (2011). Seguridad social y empleo informal. Disponible en: http://www.dane.gov.co.

Faria, V. (1976). Occupational marginaly, Employement and poverity in Brazil. Harvard University Massachussetts (EE. UU.).

Farias, A. H. (2012). La marginalidad socio-laboral: revisita de un debate desde nuevos anclajes. Un estudio de los partidos del Gran Buenos Aires 1992 - 2007. Argumentos. Revista de Crítica Social No 14116 - 148.

Flórez, C. (2002). The function of urban informal sector employment: evidence from Colombia 1984- 2000. Documento CEDE.

Grupo de Investigación Economía Social (GIES). (2005). Apuntes sobre la economía informal. Medellín: Universidad de Medellin.

López, H (2009). Economistas colombianos. Disponible en: http://www. banrepcultural.org/blaavirtual/ayudadetareas/economia/econo71.htm

Ministerio de Trabajo. (2009). Bogotá: El Ministerio. Disponible en: http://www. global-labor.org/la_economia_informal.htm.

Mondragón-Vélez C, Peña X, Wills D (2009). Labor market rigidities and informally in Colombia. Universidad de los Andes: Mimeo.

Núñez, J. (2002). Empleo informal y evasión fiscal en Colombia. DNP: archivo de economía (210).

Ochoa, V. (2005). Economía informal: Evolución reciente y perspectivas. Disponible en: http://www.diputados.gov.mex.

OIT. (1972). El dilema del sector no estructurado. Memoria del Director General. Conferencia Internacional del Trabajo. 78 Reunión. 
PREALC. (1993). PREALC: 25 Years. Santiago de Chile: Ograma.

Santa María, M. (2009). Análisis cuantitativo y cualitativo de la informalidad empresarial en Colombia. Desarrollo y Sociedad No 63 269-296.

Uribe, J. C. y Ortiz, C. (2004). Determinantes de la decisión en el mercado laboral: la decisión de ser informal en Colombia 1988 - 2000. Documento de Trabajo CIDSE (002321). 